Article

\title{
Genome-Wide Analysis of Coding and Long Non-Coding RNAs Involved in Cuticular Wax Biosynthesis in Cabbage (Brassica oleracea L. var. capitata)
}

\author{
Xiaowei Zhu ${ }^{\dagger}$, Xiang Tai ${ }^{\dagger}$, Yunying Ren, Jinxiu Chen ${ }^{*}$ and Tianyue Bo ${ }^{*}$ \\ Horticulture Research Institute, Shanghai Academy of Agricultural Sciences, Shanghai Key Lab of Protected \\ Horticultural Technology, Shanghai 201403, China; xiaoweizhusaas@163.com (X.Z.); 555666tx@163.com (X.T.); \\ yy11@saas.sh.cn (Y.R.) \\ * Correspondence: cabbagecjx@126.com (J.C.); tybo@saas.sh.cn (T.B.); Tel.: +86-189-3996-2381 (J.C.); \\ +86-189-1816-2190 (T.B.) \\ † These authors contributed equally to this work.
}

Received: 29 April 2019; Accepted: 7 June 2019; Published: 10 June 2019

\begin{abstract}
Cuticular wax is a mixture of very long chain fatty acids (VLCFAs) and their derivatives, which determines vital roles for plant growth. In cabbage, the cuticular wax content of leaf blades is an important trait influencing morphological features of the head. Understanding the molecular basis of cuticular wax biosynthesis can help breeders develop high quality cabbage varieties. Here, we characterize a cabbage non-wax glossy (nwgl) plant, which exhibits glossy green phenotype. Cryo-scanning electron microscope analysis showed abnormal wax crystals on the leaf surfaces of $n w g l$ plants. Cuticular wax composition analyzed by GC-MS displayed severely decreased in total wax loads, and individual wax components in $n w g l$ leaves. We delimited the NWGL locus into a 99-kb interval between the at004 marker and the end of chromosome C08 through fine mapping. By high-throughput RNA sequencing, we identified 1247 differentially expressed genes (DEGs) and 148 differentially expressed lncRNAs in $n w g g l$ leaves relative to the wild-type. Gene Ontology (GO) and Kyoto Encyclopedia of Genes and Genomes (KEGG) pathway enrichment analysis revealed that the DEGs and cis-regulated target genes for differentially expressed lncRNAs were significantly enriched in wax and lipid biosynthetic or metabolic processes. Our results provide the novel foundation to explore the complex molecular basis of cuticular wax biosynthesis.
\end{abstract}

Keywords: cabbage; $n w$ gl phenotype; fine mapping; cuticular wax biosynthesis; RNA-seq; differential expressed genes; IncRNAs

\section{Introduction}

The aerial surface of land plants is covered with a hydrophobic cuticle layer, which is composed of two major lipid components, the cutin polyester and the non-polymerized cuticular waxes. Cuticular waxes are predominantly composed of very long chain fatty acids (VLCFAs) and their derivatives, including aldehydes, alcohols, alkanes, ketones, and esters [1,2]. The biosynthesis of cuticular wax begins with de novo synthesized $\mathrm{C} 16$ and $\mathrm{C} 18$ fatty acids, which are then elongated into VLCFAs by a fatty acid elongase (FAE) complex, consisting of $\beta$-ketoacyl-CoA synthase, $\beta$-ketoacyl-CoA reductase, $\beta$-hydroxyacyl-CoA dehydratase, and enoyl-CoA reductase. The formed VLCFAs are further converted into various derivatives via two independent pathways, the alkane-forming and alcohol-forming pathways [3,4]. Cuticular wax protects plants against numerous biotic and abiotic stresses, such as pathogen infection and non-stomatal water loss [5,6]. It also plays important roles in 
normal plant developmental processes, including the pollen-pistil interaction [7]. Therefore, identifying plants with cuticular wax deficiency and elucidating the regulatory mechanisms controlling cuticular wax biosynthesis will be beneficial for improving agricultural crops.

To date, many genes associated with cuticular wax biosynthesis pathway have been isolated and characterized in different plant species. For example, CER1 encodes an alkane-forming enzyme and may physically interact with CER3 and cytochrome b5 isoforms (CYTB5s) to catalyze the synthesis of very long chain alkanes $[8,9]$. CER4 is an alcohol-forming fatty acyl-coenzyme A reductase (FAR) in the synthesis of primary alcohols [10]. In addition, several APETALA2/ETHYLENE-RESPONSIVE FACTOR (AP2/EFR) type transcription factors have also been reported to be involved in cuticular wax biosynthesis through regulating the expression of wax biosynthesis genes [11-17]. For instance, WRINKLED4 (WRI4) activates the expression of LACS1, KCR1, PAS2, ECR, and WSD1 by direct binding to their promoters [16].

In recent years, several RNA transcripts with no protein coding potential have been reported that have a key role in regulation of cuticular wax biosynthesis. These noncoding RNAs include some small RNA species, such as small interfering RNA (siRNA) and microRNA (miRNA), mediated silencing transcription of the wax biosynthetic genes [18-22]. Cuticular wax biosynthesis also involves long noncoding RNAs (lncRNAs). LncRNAs are a class of non-coding RNA transcripts with a length of more than $200 \mathrm{nt}$. In wheat, INHIBITOROFWAX1 (Iw1) and its homolog Iw2 serve as miRNA precursors that produce an miRNA, miRW1, which represses the expression of the putative carboxylesterase genes that are necessary for $\beta$-diketone deposition in wheat [22].

For cabbage, several cuticular wax deficiency mutants have been reported. The phenotype of 10Q-961 and g21-3 mutants is controlled by a same recessive locus, which was finely mapped to chromosome C08 through classical linkage analysis $[23,24]$. A dominant 10Q-974gl mutant gene BoGL1 was also fine mapped to chromosome C08 [25]. Moreover, both the BoWAX1 and Cgl2 genes were mapped to chromosome C01 [26,27]. In addition, the $C g l 2$ gene in cabbage had been cloned and could rescue the phenotype of the Arabidopsis cer4 mutant [27]. However, the cuticular wax biosynthesis genes have not been fully explored in cabbage.

In this study, we report the phenotypic and characterization of the non-wax glossy (nwgl) plant characterized by a glossy appearance of the cabbage. The $n w g l$ plants exhibited a marked reduction in amounts of total wax and changes in wax components. We performed fine mapping and delimited the NWGL gene responsible for the $n w g l$ phenotype to the end of chromosome C08. By conducting RNA-seq, we identified differential expressed genes (DEGs) and lncRNAs in leaf tissues of $n w g g l$ and wild-type plants. Moreover, we predicted both cis- and trans- regulatory target genes for the differential expressed lncRNAs. Functional analysis of the DEGs and cis-regulated targets genes for differential expressed IncRNAs revealed significantly enriched categories and pathways related with wax and lipid biosynthesis and/or metabolism, providing novel implication underlying cuticular wax biosynthesis in cabbage.

\section{Results}

\subsection{Characterization of the Plant Exhibiting nwgl Phenotype}

The plant exhibiting $n w g l$ phenotype was identified from the inbred line G287. The prominent phenotype of $n w g l$ plants was their altered wax coatings, resulting in light green leaves, stems, and flower buds (Figure 1a-f). The plant was designated nwgl based on its non-wax and glossy appearance. Cryo-scanning electron microscopy analysis revealed that the cuboid wax crystals were distributed densely on the leaf surface of the wild-type, whereas only fewer granular wax crystals were deposited on the leaf surface of $n w g l$ plants (Figure 1g,h). Therefore, these results indicate that the $n w g l$ mutation might cause abnormalities in specific components of the cuticular wax of cabbage plants. 

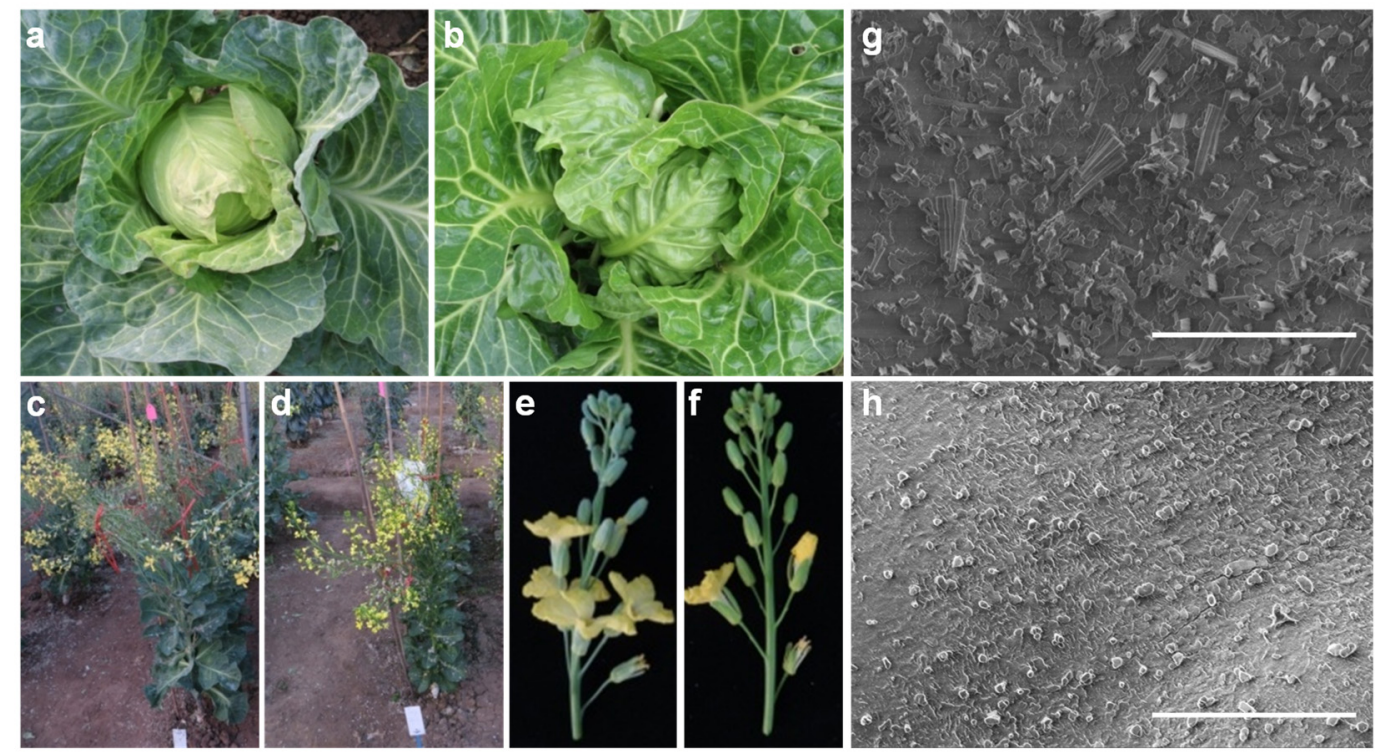

Figure 1. Phenotypes of wild-type and $n w g l$ plants. The leaf appearance of wild-type (a) and $n w g l$ plants (b) at the head harvesting stage. Wild-type (c) and nwgl (d) plants at the flowering stage. (e) Wild-type flowers. (f) $n w g l$ flowers. Cuticular wax crystals formed on adaxial surface of the wild-type (g) and $n w g l(\mathbf{h})$ leaf blades visualized by cryo-scanning electron microscopy. Bar $=10 \mu \mathrm{m}$ in (g) and (h).

\subsection{Cuticular Wax Composition of nwgl Plants}

To investigate why the plants exhibiting nwgl phenotype had a significant depletion in wax crystals, we examined the content and the constitution of cuticular waxes from leaf blades of $n w g l$ and wild-type plants by gas chromatography-mass spectrometry (GC-MS). Compared with wild-type plants, the total amount of all wax loads on $n w g l$ leaf blades was reduced by $90.7 \%$ (Table 1 ). The contents of VLCFA, alkanes, and primary alcohols decreased by $45.8 \%, 98.3 \%$, and $42.5 \%$ in $n w g l$ leaf blades, respectively (Table 1). The secondary alcohol and ketone were undetectable in $n w g l$ leaf blades (Table 1). However, the content of aldehyde exhibited slightly increased in $n w g l$ leaf blades (Table 1). Further analysis revealed significantly reduced contents of C26 VLCFA, C27 and C29 alkanes, and C28 primary alcohol. Meanwhile, neither C28 secondary alcohol nor C29 ketone was detected in $n w g l$ leaf blades (Figure 2). Taken together, these findings suggest that the reduced wax crystals in $n w g l$ leaf blades result from decreased levels of VLCFAs and their individual derivatives.

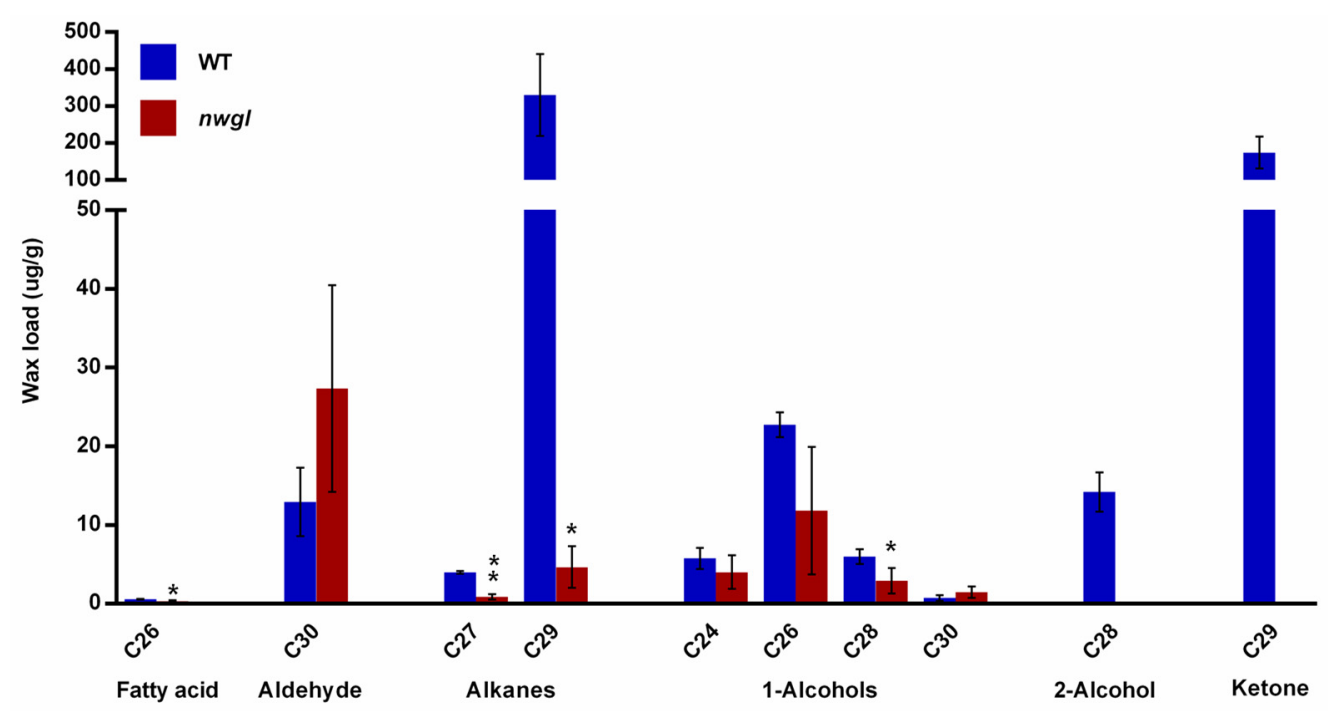

Figure 2. Cuticular wax composition in leaves from wild-type and $n w g l$ plants. Error bars indicate SD $(n=3) .{ }^{*} p<0.05 ;{ }^{* *} p<0.01$. 
Table 1. Cuticular wax composition of leaf blades from wild-type and nwgl plants.

\begin{tabular}{ccc}
\hline Components & Wild-type $(\mu \mathrm{g} / \mathrm{g})$ & $n w g l(\mu \mathrm{g} / \mathrm{g})$ \\
\hline Fatty acid & $0.59 \pm 0.04$ & $0.32 \pm 0.14^{*}$ \\
Aldehyde & $12.93 \pm 4.33$ & $27.33 \pm 13.15$ \\
Alkanes & $334.36 \pm 110.66$ & $5.52 \pm 2.97 *$ \\
1-Alcohols & $35.19 \pm 2.74$ & $20.23 \pm 12.52$ \\
2-Alcohol & $14.20 \pm 2.49$ & - \\
Ketone & $174.06 \pm 43.09$ & - \\
Total & $571.33 \pm 152.22$ & $53.40 \pm 28.31^{* *}$ \\
\hline
\end{tabular}

Data are mean $\pm \operatorname{SD}(n=3) .{ }^{*} p<0.05,{ }^{* *} p<0.01$; Student's $t$-test.

\subsection{Fine Mapping of NWGL}

Genetic analysis showed that the $n w g l$ phenotype is a dominant mutation controlled by a single gene, with one quarter of the $\mathrm{F}_{2}$ progeny displaying a normal waxy appearance $\left(208: 61, \chi^{2}=0.67\right.$, $\left.p>0.05 ; 270: 91, \chi^{2}=0.04, p>0.05\right)$. To isolate the NWGL gene, we generated two $\mathrm{F}_{2}$ mapping populations by crossing plants exhibiting $n w g l$ phenotype with two wild-type inbred lines G306 and G274, respectively. The NWGL locus was mapped preliminarily between simple sequence repeat (SSR) marker at 040 and the end of chromosome C08 (Figure 3). Using newly developed InDel and SSR markers, the NWGL gene was fine-mapped into a 99-kb DNA segment between marker at004 and the end of chromosome C08 (Figure 3). There were 16 putative open reading frames (ORFs) within the 99-kb genomic region according to the cabbage gene annotation database (www.ocri-genomics.org/bolbase, accessed on: 9 June 2019) (Figure 3). Except for Bol018503 and Bol018504, the functions of these genes are either unknown or do not involve in cuticular wax biosynthesis (Table S1). Bol018503 encodes a CER1-L1 homolog, which belongs to fatty acid hydroxylase superfamily in Arabidopsis, and Bol018504 encodes an aldehyde decarbonylase (CER1), which is known to promote wax very long chain alkane biosynthesis in various plant species $[8,9,28,29]$. To define which of these carries the $n w g g l$ mutation, we sequenced the two candidate genes, including their $5^{\prime}$ and $3^{\prime}$ flanking sequences, but no DNA sequence alteration was found. However, examination of gene expression of all the 16 ORFs within the fine mapping region only showed a down-regulation of Bol018504 in $n w g l$ leaves but not in the wild-type, indicating that the decreased expression of Bol018504 might cause the nwgl phenotypes (Figure $4 \mathrm{~b}$, Table S2).This speculation was in accordance with a recent study, in which the same expression abundance decrease of Bol018504 was identified in an investigation of a dominant glossy green cabbage mutant 10Q-974gl [25].

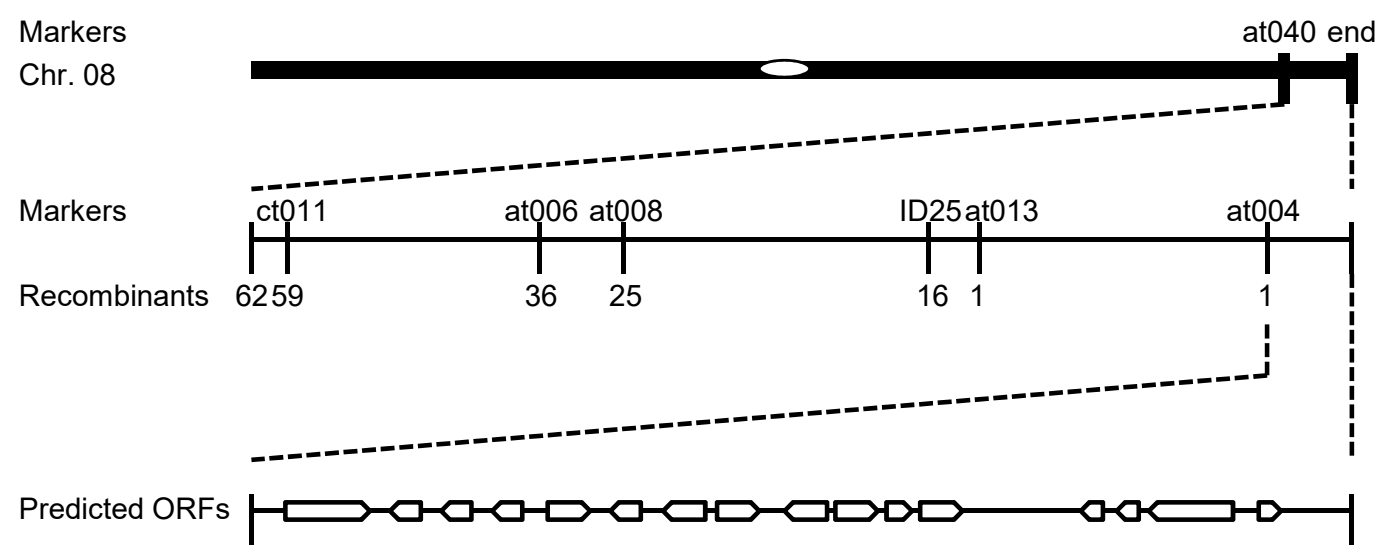

Figure 3. Fine mapping of NWGL. The NWGL locus was mapped onto chromosome C08. Molecular markers and ORFs in the mapping region are shown. 
a

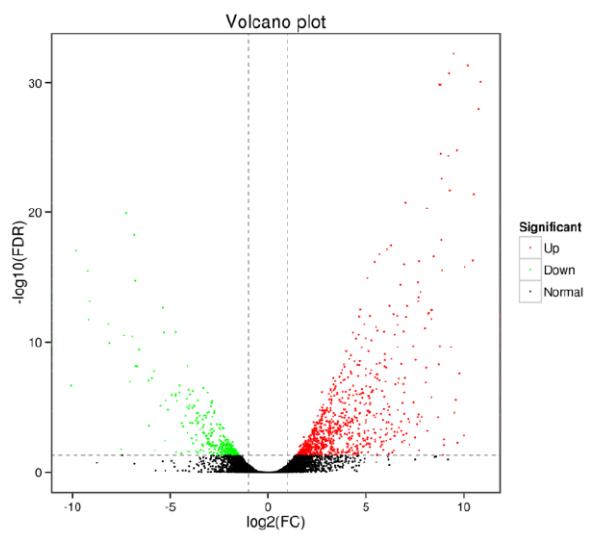

b
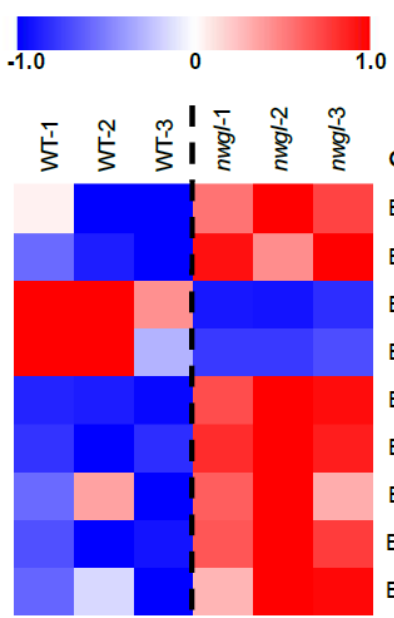

\begin{tabular}{|c|c|}
\hline ene id & Description \\
\hline 3ol025251 & aldehyde decarbonylase \\
\hline Bol025577 & aldehyde decarbonylase \\
\hline 3ol018504 & aldehyde decarbonylase \\
\hline ol013612 & Long-chain-fatty-acyl-CoA reductase \\
\hline 1035700 & Long-chain-fatty-acyl-CoA reductase \\
\hline 01036039 & Long-chain-fatty-acyl-CoA reductase \\
\hline 01024192 & 3-ketoacyl-CoA synthase \\
\hline 1038193 & AP2-like factor \\
\hline 1038557 & AP2-like factor \\
\hline
\end{tabular}

Name Function

CER1 Wax biosynthesis

CER1 Wax biosynthesis

CER1 Wax biosynthesis

CER4 Wax biosynthesis

Suberin/Wax biosynthesis

Suberin/Wax biosynthesis

Fatty acid elongation

Wax biosynthesis

Wax biosynthesis

c

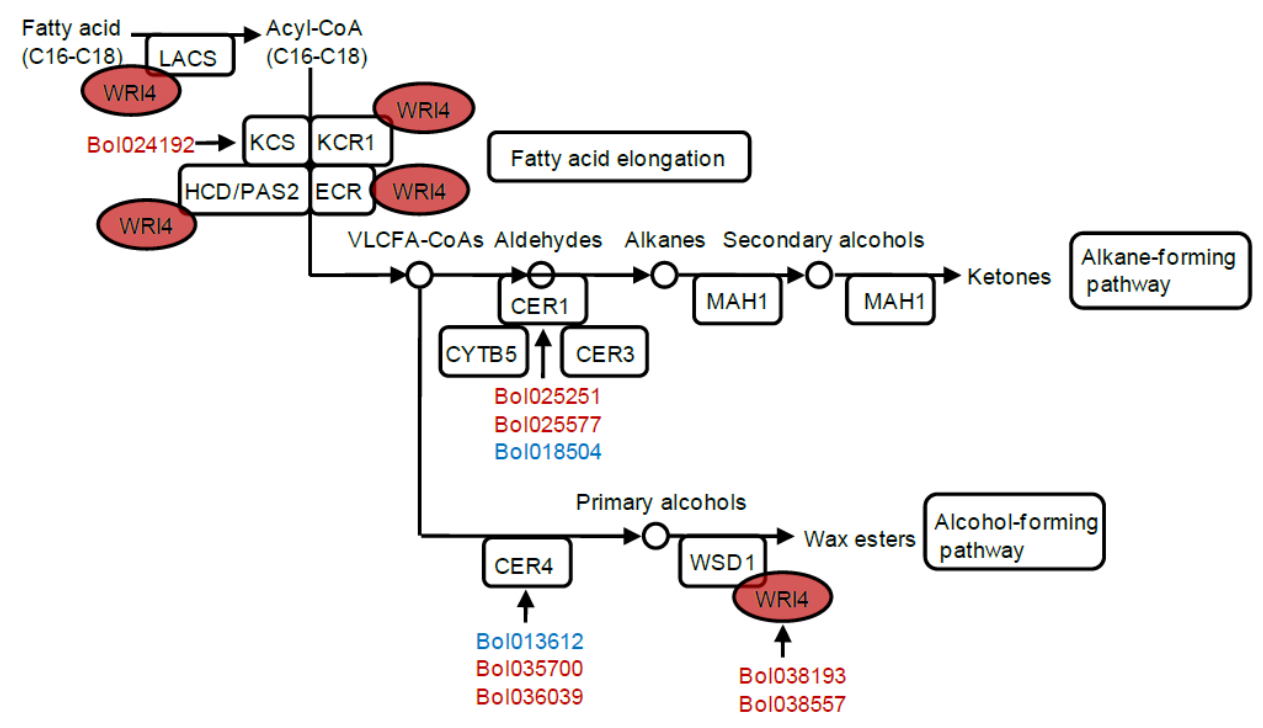

Figure 4. Analysis of DEGs in the RNA-seq libraries. (a) Volcano plot showing significant DEGs. Red and green dots represent up and down-regulated DEGs, respectively (FDR <0.05). Black dots are genes that were not differently expressed. (b) Gene expression of DEGs involved in wax biosynthesis. The data are shown in base 2 logarithmic form (log2 FPKM). Three biological replicates of wild-type (WT) and nwgl leaves are exhibited. (c) Simplified pathways for wax biosynthesis. LACS, long chain acyl-CoA synthetase; KCS, $\beta$-ketoacyl-CoA synthase; KCR, $\beta$-ketoacyl-CoA reductase; HCD/PAS2, $\beta$-hydroxyacyl-CoA dehydratase; ECR, enoyl-CoA reductase; CER, ECERIFERUM; CYTB5, cytochrome b5 isoform; MAH, mid-chain alkane hydroxylase; WSD, wax synthase/diacylglycerol acyltransferase; WRI4, WRINKLED4. Down-regulated and up-regulated genes in $n w g l$ vs. wild-type plants are marked in green and red color, respectively. 
These results suggest that the Bol018504 might be regulated by the NWGL locus or other mechanisms, such as an epigenetic manner. We further investigated the DNA methylation level of Bol018504 by employing three methylation sensitive restriction enzymes. For McrBC-PCR analysis, we found that the middle region of Bol018504 corresponding to the fragment " $\mathrm{C}$ " (+905 to +1615$)$ was unmethylated in the wild-type but slightly methylated in nwgl plants (Figure S1a,b).The HpaII, MspI-PCR analysis was used to detect the methylation level of CCGG sites within fragments " $\mathrm{A}$ " $(-1113$ to -408$)$, "B" $(+189$ to +1069$)$, and " $C$ " $(+905$ to +1615$)$. The results indicated that the $C$ of the CCGG sites within fragments "B" $(+189$ to +1069$)$ and "C" $(+905$ to +1615$)$ might be hypermethylated in $n w g l$ plants compared with the wild-type (Figure S1a,c).

\subsection{RNA-seq Analysis of Plants Exhibitingnwgl Phenotype}

To further explore the global impacts of the $n w g l$ mutation which could reduce cuticular wax contents in plants exhibiting $n w g l$ phenotype, we conducted a transcriptome comparison of $n w g l$ and wild-type leaves by high-throughput RNA sequencing (RNA-seq). In total, 1247 protein coding genes were differentially expressed in $n w g l$ leaves relative to wild-type, including 913 up-regulated genes and 334 down-regulated genes (Figure 4a, Table S2).

We then performed Gene Ontology (GO) analysis to assess the function of the DEGs. Notably, the down-regulated genes most significantly enriched were in the wax biosynthetic process (GO: 0010025), while the up-regulated genes most significantly enriched were in response to chitin (GO: 0010200) (Tables S3 and S4). In addition, a significant fraction of up-regulated genes were involved in lipid-related GO categories, including lipid localization (GO: 0010876), positive regulation of fatty acid metabolic/biosynthetic process (GO: 0045923 and GO: 0045723), glycerolipid metabolic process (GO: 0046486), neutral lipid metabolic process (GO: 0006638) and sphingolipid catabolic process (GO: 0030149) (Table S4), suggesting that NWGL might be involved in wax biosynthesis through regulating the expression of lipid-related genes.

To further understand the metabolic pathways associated with NWGL, the DEGs were mapped to the KEGG database to analyze the enriched pathways. There were nine pathways highly enriched for down-regulated genes, and the most significantly enriched encoded proteins were involved in 'tyrosine metabolism' (KEGG ID: ko00350) (Table S5). The 'cutin, suberine and wax biosynthesis' (KEGG ID: ko00073), 'linoleic acid metabolism' (KEGG ID: ko00591), and 'sphingolipid metabolism' (KEGG ID: ko00600) were significantly enriched pathways in up-regulated genes (Table S6), supporting the notion that NWGL functions in wax and lipid metabolism.

When focusing on the wax biosynthetic process, nine genes showed an altered expression in $n w g l$ leaves relative to the wild-type. Only two genes associated with wax biosynthesis were down-regulated in nwgl leaves (genes encoding CER1 (Bol018504) and CER4 (Bol013612)), while seven genes were up-regulated: genes encoding another two CER1 proteins (Bol025251 and Bol025577), two Long-chain-fatty-acyl-CoA reductases (Bol035700 and Bol036039), one 3-ketoacyl-CoA synthase (Bol024192) and two AP2-like factors (Bol038193 and Bol038557) (Figure 4b,c).

\subsection{Identification and Characterization of $\operatorname{lnc} R N A$ s in Cabbage}

To screen out potential lncRNAs involved in regulating wax biosynthesis, we thoroughly analyzed the cabbage noncoding transcriptome and identified 4459 putative lncRNAs (Figure 5a, Table S7). The 4459 lncRNA sequences were compared with mRNAs obtained in this study. These lncRNAs were shorter than mRNAs, $76.3 \%$ of which being $<1000 \mathrm{bp}$ in length (Figure $5 \mathrm{~b}$ ). Most lncRNAs $(88.0 \%)$ had two or three exons in their transcripts and were fewer than protein-coding transcripts (Figure 5c). Interestingly enough, the two features of lncRNAs in cabbage, shorter lengths and fewer exons, have also been detected in other Brassica species, including Chinese cabbage, rapeseed, kale, and non-heading Chinese cabbage [30-32]. Moreover, lncRNAs also had shorter ORFs compared with mRNAs, with approximately $92.2 \%$ at $<100$ amino acids (Figure $5 d$ ). 
a

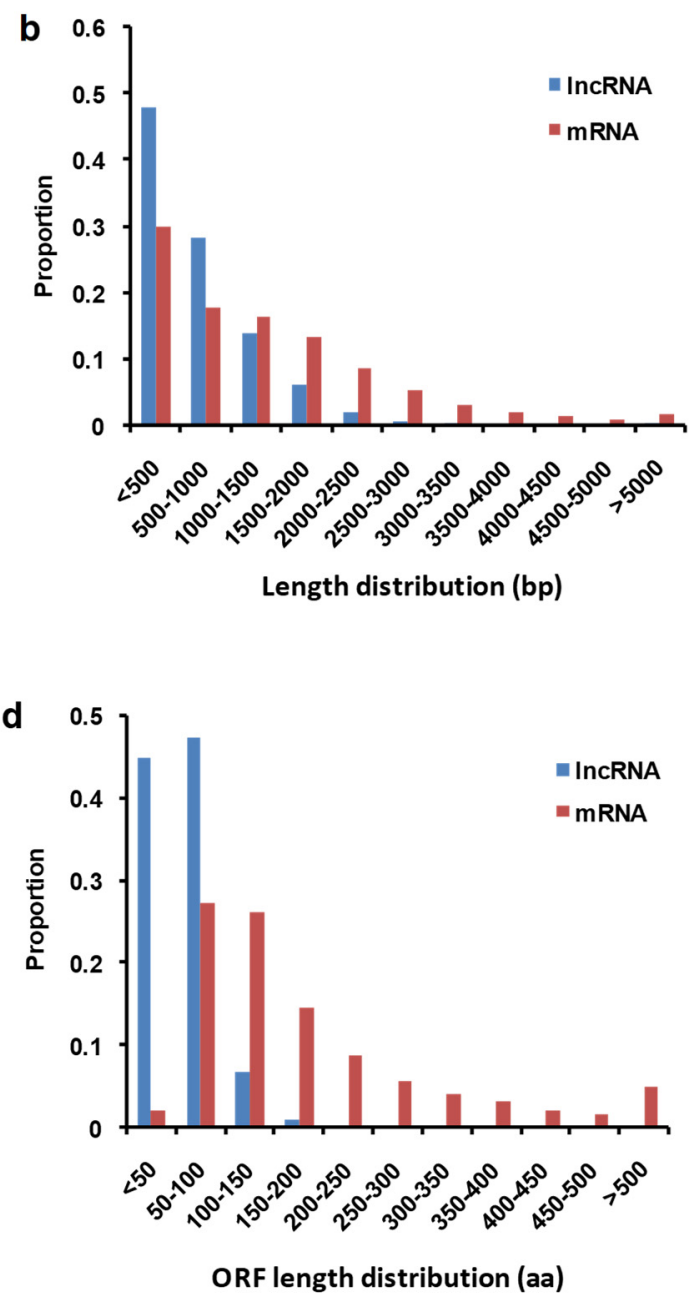

Figure 5. Characteristics of lncRNAs identified in cabbage. (a) The 4459 lncRNAs in cabbage were identified by an intersection of results obtained via three softwares CNCI (Coding-Non-Coding Index), CPC (Coding Potential Calculator), CPAT (Coding Potential Assessment Tool) and a protein database Pfam analysis. (b) Length distribution (base pair, bp) of lncRNAs and mRNAs. (c) Exon number in lncRNAs and mRNAs. (d) ORF length in amino acids (aa) of lncRNAs and mRNAs.

\subsection{Identification of Differentially Expressed IncRNAs between nwgl and Wild-Type Plants}

A total of 148 lncRNAs were found to be significantly differentially expressed (Figure 6a, Table S8). In detail, 96 lncRNAs in $n w g l$ leaves were up-regulated and 52 were down-regulated compared with the wild-type (Figure 6a, Table S8). LncRNAs have been shown to regulate protein coding gene expression via cis- and trans-action [33]. In total, 929 annotated target genes for 148 differentially expressed lncRNAs were predicted, of which 906 were cis-regulatory and 23 were trans-regulatory (Tables S9 and S10). Gene Ontology (GO) terms were identified for the 906 cis-regulated target genes (Figure 6b). The top enriched GO terms in the biological process, cellular component, and molecular function categories were organic cyclic compound biosynthetic process (GO: 1901362), nucleus (GO: 0005634), and protein binding (GO: 0005515), respectively (Table S11). In addition, the GO term glycerolipid metabolic process (GO: 0046486) was highly enriched (Table S11). Moreover, the 906 cis-regulated target genes were significantly enriched in KEGG pathways, consisting of the pentose phosphate pathway (KEGG ID: ko00030), endocytosis (KEGG ID: ko04144), ribosome (KEGG ID: ko03010), glycolysis/gluconeogenesis (KEGG ID: ko00010), degradation of aromatic compounds (KEGG ID: ko01220), and oxidative phosphorylation (KEGG ID: ko00190) (Table S12).

To further analyze the function of differentially expressed lncRNAs, we investigated the co-expression correlation between differentially expressed lncRNAs and their predicted target genes. 
A total of 206 interaction relationships (111 positive and 95 negative correlations) were discovered between 97 lncRNAs and 163 target genes (Table S13). Notably, some of the correlated target genes were involved in lipid-related processes, including fatty acid metabolic process, fatty acid beta-oxidation, fatty acid degradation, and fatty acid homeostasis (Figure 6c).

a



b

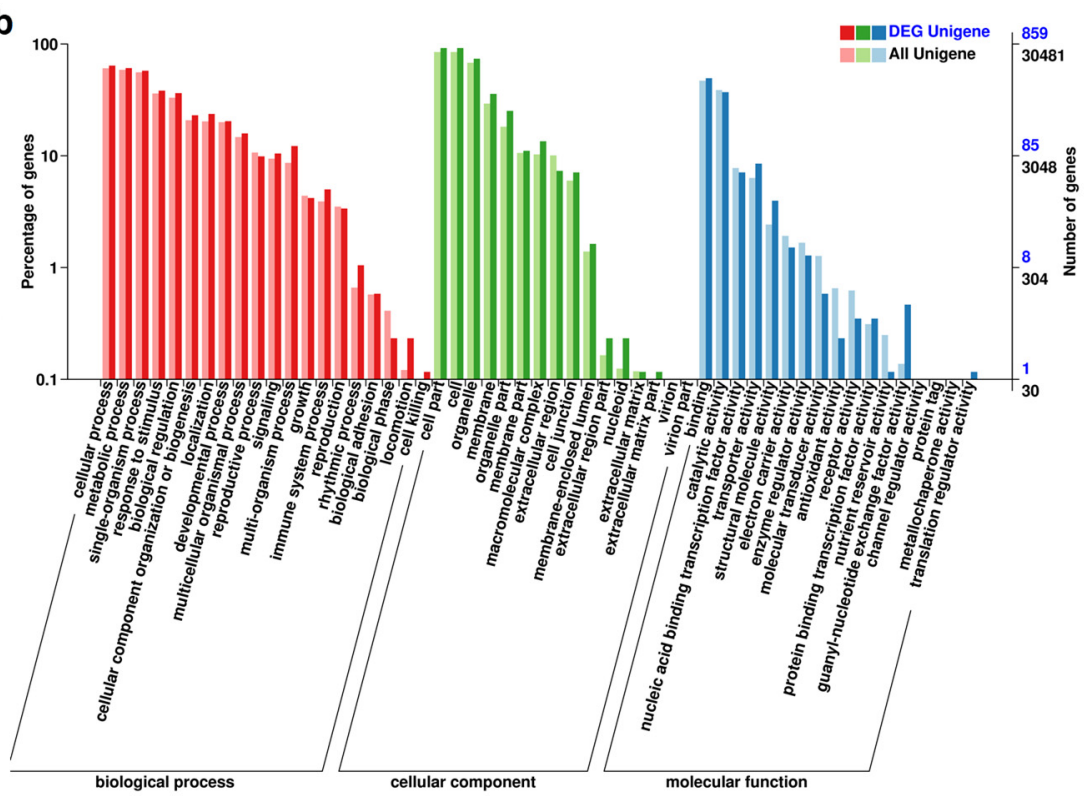

C

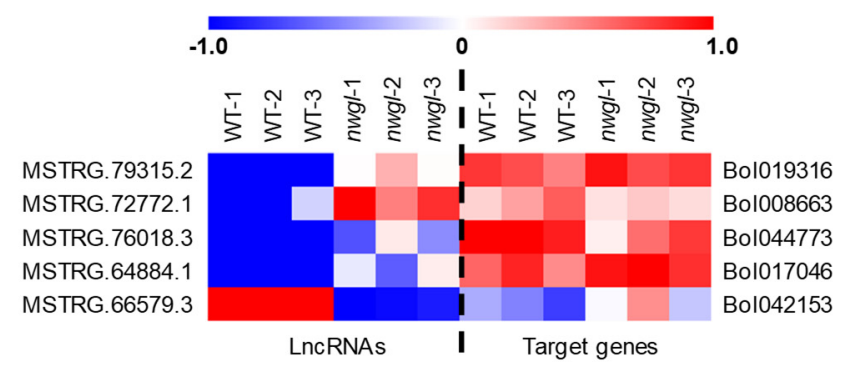

Figure 6. Differentially expressed lncRNAs in $n w g l$ vs. wild-type plants. (a) Hierarchical clustering analysis of the differentially expressed lncRNAs. The data are expressed in base 10 logarithmic form ( $\log 10$ FPKM+0.000001). (b) GO classification of 906 cis-regulated target genes of differentially expressed lncRNAs. (c) The co-expression differentially expressed lncRNAs and target genes involved in lipid-related processes. The data are exhibited in base 2 logarithmic form (log2 FPKM). Three biological replicates of wild-type (WT) and nwgl plants are shown.

\section{Discussion}

The content of cuticular waxes is an important agronomic trait for cabbage breeding. However, there are only a few reports regarding wax biosynthesis in cabbage [23-27]. The phenotype and characterization of the nwgl plants reported here showed that NWGL is a factor for cuticular wax biosynthesis in cabbage. The nwgl leaf blades exhibited decreased contents of VLCFA, alkanes and primary alcohols, and the abortion of secondary alcohol and ketone (Table 1), indicating that NWGL acted as an major regulator for VLCFA elongation and the two distinct pathways, the alkane-forming and alcohol-forming pathways. The involvement of NWGL in the synthesis of various wax compounds was reconfirmed by the RNA-seq analysis. Firstly, GO and KEGG pathway analysis showed that DEGs between plants exhibiting $n w g l$ phenotype and the wild-type were enriched in categories related to wax and lipid synthesis/metabolism (Tables S3-S6). Secondly, nine DEGs which encode proteins involved in VLCFA production, alkane-forming and alcohol-forming pathways of wax biosynthesis are regulated by NWGL (Figure 4c). Thirdly, five of the target genes that were found to be strongly co-expressed 
with the differentially expressed lncRNAs were annotated to be involved in lipid-related processes (Figure 6c). Collectively, our data suggest that NWGL is involved in multiple wax biosynthetic processes, from upstream fatty acid synthesis to subsequent wax production.

Our results demonstrated that the glossy phenotype of the nwgl plants is determined by a single dominant locus within approximately $99-\mathrm{kb}$ between at004 and the end of chromosome C08 (Figure 3). Therefore, these results indicated that the NWGL gene may be an allele of the previously studied BoGL1 gene [25]. Unfortunately, we failed to identify DNA lesion of Bol018504 in nwgl plants, except its reduced expression abundance and altered DNA methylation level, which was located in the fine mapping region and encodes an alkane-forming enzyme (BoCER1). Previous studies have reported that the Arabidopsis thaliana CER1 protein is an essential element of wax alkane synthesis [8,9]. The Arabidopsis cer1 mutant exhibited a dramatic decrease in alkanes and nearly depleted of secondary alcohols and ketones, accompanied by a slight increase in aldehyde content as compared to wild-type plants [8,34-36]. Interestingly, our GC-MS analyses found significant reductions in total wax in $n w g l$ leaf blades, especially for C27 and C29 alkanes, and the undetected C28 secondary alcohol and C29 ketone, indicating that Bol018504 played a critical role in NWGL-regulated cuticular wax biosynthesis. However, we cannot rule out the possibility that other unknown genes within the fine mapping region may be responsible for NWGL, which may prove another interesting area for further investigation. For instance, the VLCFA component, which cannot be catalyzed by CER1, also decreased significantly in $n w g l$ leaf blades.

The interaction of NWGL with other genes was investigated via transcriptome analysis. Based on our RNA-seq data, the expression of nine genes, whose homologs in Arabidopsis thaliana are key factors involved in wax biosynthesis, was down-regulated or up-regulated in $n w g l$ leaves $[8,10,16,37,38]$. Except for Bol018504, the expression of Bol013612 was down-regulated in nwgl leaves. Bol013612, which encodes fatty acyl-CoA reductase, is homologous to Arabidopsis thaliana CER4 [10]. The wax components of the cabbage Bol013612 loss-of-function mutant were lacking in primary alcohols and wax esters [27]. Moreover, the $n w g l$ leaf blades showed significant reduction in C28 primary alcohol, suggesting that the effect of NWGL on wax biosynthesis might require Bol013612 at the mRNA level. However, the expression levels of five genes, which encode one 3-ketoacyl-CoA synthase (Bol024192), another two CER1 proteins (Bol025251 and Bol025577), and two fatty-acyl-CoA reductases (Bol035700 and Bol036039), were up-regulated in $n w g l$ leaves. These results indicated that the altering wax amount in the $n w g l$ mutant resulted in feedback, causing up-regulation of some genes in fatty acid elongation, alkane-forming, and alcohol-forming pathways of wax biosynthesis. In addition, transcriptional control is considered to be the major mechanism for determining the total wax deposition in Arabidopsis thaliana [2]. The remaining two up-regulated genes-Bol038193 and Bol038557-in $n w g l$ leaves, which encode AP2-like factors, are $81.2 \%$ and $88.0 \%$ homologous to Arabidopsis thaliana WRI4 transcription factor, respectively. WRI4 is a transcriptional activator and functions in the up-regulation of cuticular wax biosynthesis in Arabidopsis stems [16]. Therefore, these findings suggest that the up-regulated genes in $n w g l$ leaves might be controlled by NWGL through WRI4 transcription factor.

Although the number of known plant lncRNAs is increasing dramatically, the identification of lncRNAs as regulators in cuticular wax biosynthesis in plants is still lacking [22,30-32,39-46]. In this study, we identified 148 differentially expressed lncRNAs between plants exhibiting nwgl phenotype and wild-type (Figure 6a, Table S8), revealing specific responses of the noncoding transcriptome to cuticular wax loads reduction. The fact that these lncRNAs changed expression pattern in $n w g l$ leaves suggested that at least some of them have regulatory roles in wax biosynthesis. LncRNAs can regulate gene expression by cis- and trans-acting [33]. We predicted the cis-regulated and trans-regulated target genes of the differentially expressed lncRNAs and performed a co-expression analysis between them to explore the putative regulatory functions of the lncRNAs. Five of the target genes, which were found to be strongly co-expressed with the differentially expressed lncRNAs, were annotated to be involved in lipid-related processes. Unfortunately, we did not find any target genes of the differentially expressed 
IncRNAs directly involved in cuticular wax biosynthesis. It would be difficult, but worthwhile, to investigate the exact roles of these differentially expressed lncRNAs in cuticular wax biosynthesis.

In summary, we fine-mapped the NWGL locus underlying the non-wax glossy phenotype in cabbage. Through genome-wide transcriptome studies, we revealed that many protein-coding genes involved in wax biosynthesis were regulated by the NWGL gene. Perhaps the most striking finding by this work was genome-wide identification of a set of lncRNAs differentially expressed in plants exhibiting $n w g l$ phenotype, and may reveal a more sophisticated molecular regulation mechanism of cuticular wax biosynthesis in cabbage.

\section{Materials and Methods}

\subsection{Plant Materials}

The cabbage (Brassica oleracea L. var. capitata) plant exhibiting (non-wax glossy) nwogl phenotype was derived from inbred line G287. Two $\mathrm{F}_{2}$ mapping populations were generated by crossing the plant exhibiting nwgl phenotype with inbred line G306 or G274, respectively. Cabbage plants were cultivated in the experimental field at the Shanghai Academy of Agricultural Sciences (Shanghai, China) during the natural growing season.

\subsection{Cryo-scanning Electron Microscopy (Cryo-SEM)}

Fresh leaf samples from $n w g l$ and wild-type plants were fixed in specimen holders of a Hitachi S4800 cryo-transfer system (Hitachi, Tokyo, Japan) with glue, immediately frozen with liquid nitrogen. The samples were transferred to a preparation chamber under vacuum for coating. Photographs of the sample surface were taken using its carrying camera.

\subsection{Cuticular Wax Analysis}

For the wax composition studies, cabbage rosette leaf segments were collected at the harvesting stage and immersed in liquid nitrogen for storage. For wax extraction, 200-400 mg of freeze-dried samples were dipped into $15 \mathrm{~mL}$-hexane for $30 \mathrm{~s}$ at $60{ }^{\circ} \mathrm{C}$ and dried under a stream of nitrogen. By adding $100 \mu \mathrm{L}$ n-hexane, $100 \mu \mathrm{L} \mathrm{N}$,O-bis (trimethylsilyl) fluoroacetamide (BSTFA), and $2 \mu \mathrm{L}$ n-tetracosane $(20 \mathrm{mg} / \mathrm{mL})$ as an internal standard, samples were shocked for $1 \mathrm{~min}$ and subsequently incubated for $30 \mathrm{~min}$ at $90{ }^{\circ} \mathrm{C}$. These derivatized samples were then analyzed with an Agilent 7890B-5977B GC-MS (Agilent, Santa Clara, CA, USA).

\subsection{Fine Mapping of NWGL}

Genomic DNA was isolated from young seedlings with normal phenotype. The NWGL gene was first mapped to the end of chromosome $\mathrm{C} 08$ using $46 \mathrm{~F}_{2}$ normal individuals selected from G306×nwgl or G274×nwgl, respectively. A total of 706 recessive normal individuals selected from the $\mathrm{F}_{2}$ population $\mathrm{G} 274 \times n w \mathrm{~g} l$ were used for fine mapping. To identify candidate genes, the corresponding DNA fragments were amplified from both $n w g l$ and wild-type plants and sequenced. The primer sequences are listed in Table S14.

\subsection{RNA Isolation, Library Construction and Illumina Sequencing}

Total RNA was extracted from collected leaves of $n w g l$ and WT plants at harvesting stage using RNAprep Pure Plant kit (Polysaccarides\&Polyphenolics-rich) (Tiangen, Beijing, China) according to the manufacturer's instructions. RNA yield and purity were measured using a NanoDrop 2000 spectrophotometer (Thermo Fisher Scientific, Wilmington, DE, USA). RNA integrity was assessed with an Agilent 2100 Bioanalyser (Agilent, Santa Clara, CA, USA). Six cDNA libraries (three biological replicates per genotype) were constructed using NEBNextUltra ${ }^{\mathrm{TM}}$ Directional RNA library Prep kit (NEB, Ipswich, MA, USA) for Illumina (Illumina, San Diego, CA, USA). RNA sequencing was performed by IlluminaHiSeq platform by Biomarker Technologies Co. Ltd. (Biomarker, Beijing, China). 


\subsection{Read Mapping and Transcriptome Assembly}

Raw data were filtered using inhouse perl scripts available with Biomarker Technologies Co. Ltd. (Biomarker, Beijing, China). In total, $163.35 \mathrm{~Gb}$ clean data were generated from six libraries (on average, more than $26.48 \mathrm{~Gb}$ clean data for each sample). Clean reads were aligned to the cabbage genome (www.ocri-genomics.org/bolbase, accessed on: 13 July 2018) using HISAT 2 [47]. The mapped reads were assembled using the StringTie with gff compare program annotation [48]. The raw data were deposited in the GEO database (https:/www.ncbi.nlm.nih.gov/geo/, accessed on: 28 April 2019) and the GEO accession number is GSE130405.

\subsection{Identification of $\operatorname{lnc} R N A s$}

The unknown transcripts were used for putative lncRNA identification. The lncRNAs candidates were identified followed rigorous criteria: (1) The transcripts length must be $\geq 200 \mathrm{bp}$; (2) The transcripts must be with two or more exons; (3) The transcripts must be with FPKM (Fragments Per Kilobase of transcript per Million fragments mapped) value $\geq 0.1$. Then, the predicted lncRNAs were further screened using four computational approaches, including CPC, CNCI, Pfam and CPAT to ensure that the lncRNAs did not have protein-coding ability [49-52].

\subsection{Analysis of Differential Expression of Genes and lncRNAs}

Genes and lncRNAs expression level was estimated by calculating FPKM values using StringTie (v1.3.1) [48]. Differentially expressed genes and lncRNAs were identified using the DESeq R package (v1.10.1) with $\mid \log _{2}$ (fold change) $\mid \geq 1$ and a false discovery rate (FDR) $<0.05$. The FPKM values of genes and lncRNAs have been deposited in the GEO database (https://www.ncbi.nlm.nih.gov/geo/, accessed on: 28 April 2019) and the GEO accession number is GSE130405.

\subsection{Target Gene Prediction of $\operatorname{lnc} R N A s$}

The neighboring genes within $100 \mathrm{~Kb}$ upstream and downstream of lncRNAs on the genome were considered as potential cis-regulated target genes. The trans-regulated target genes were searched using LncTar software based on mRNA sequence complementary and RNA duplex energy prediction [53].

\subsection{Functional Enrichment Analysis}

For all the differentially expressed genes (DEGs) and cis-regulated target genes of differentially expressed lncRNAs, Gene Ontology (GO) was analyzed using topGO R packages and Kyoto Encyclopedia of Genes and Genomes (KEGG) analysis was carried out using the KOBAS software [54,55]. Significant enriched Go terms and KEGG pathways were identified with $p$ value $<0.05$.

\subsection{Co-expression Analysis}

We calculated the Spearman correlation coefficients between the expression levels of differentially expressed lncRNAs and their target genes to analyze their co-expression.

\subsection{McrBC-, HpaII, MspI-PCR}

McrBC is an endonuclease which cleaves DNA containing methylcytosine and doesnot act upon unmethylated DNA. However, McrBC cannot recognize HpaII/MspI endonuclease sites (CCGG) in which the internal cytosine is methylated. For McrBC, HpaII, MspI-PCR analysis of Bol018504, $1000 \mathrm{ng}$ of genomic DNA was digested using 20 units of the three endonucleases, respectively. The volume of enzyme reaction mixes was $50 \mu \mathrm{L}$. The reaction time was $0,0.5,3$, and $8 \mathrm{~h}$ for $\mathrm{Mcr}$ BC digestion, but 0 , and $3 \mathrm{~h}$ for HpaII and MspI digestion. The digested DNAs were used for PCR amplification, and the products were isolated by agarose gel electrophoresis. 
Author Contributions: X.Z. conceived and designed the experiments and wrote the manuscript; X.Z., X.T., Y.R., J.C., and T.B. performed the experiments and analyzed the data.

Funding: This research was funded by the Young Talent Growth Program in Agricultural System of Shanghai (2017-1-5), the Agricultural Development Program through Science and Technology of Shanghai (2015-6-1-7), the National Key Research and Development Program of China (2016YFD0101702).

Conflicts of Interest: The authors declare no conflict of interest.

\section{References}

1. Samuels, L.; Kunst, L.; Jetter, R. Sealing plant surfaces: Cuticular wax formation by epidermal cells. Annu. Rev. Plant Biol. 2008, 59, 683-707. [CrossRef]

2. Lee, S.B.; Mi, C.S. Advances in the understanding of cuticular waxes in Arabidopsis thaliana and crop species. Plant Cell Rep. 2015, 34, 557-572. [CrossRef] [PubMed]

3. Yeats, T.H.; Rose, J.K. The formation and function of plant cuticles. Plant Physiol. 2013, 163, 5-20. [CrossRef] [PubMed]

4. Bernard, A.; Joubes, J. Arabidopsis cuticular waxes: Advances in synthesis, export and regulation. Prog. Lipid Res. 2013, 52, 110-129. [CrossRef] [PubMed]

5. Weidenbach, D.; Jansen, M.; Franke, R.B.; Hensel, G.; Weissgerber, W.; Ulferts, S.; Jansen, I.; Schreiber, L.; Korzun, V.; Pontzen, R.; et al. Evolutionary conserved function of barley and Arabidopsis 3-KETOACYL-CoA SYNTHASES in providing wax signals for germination of powdery mildew fungi. Plant Physiol. 2014, 166, 1621-1633. [CrossRef] [PubMed]

6. Wang, Z.; Tian, X. The E3 ligase DROUGHT HYPERSENSITIVE negatively regulates cuticular wax biosynthesis by promoting the degradation of transcription factor ROC4 in rice. Plant Cell 2018, 30, 228-244. [CrossRef]

7. Aarts, M.G.; Keijzer, C.J.; Stiekema, W.J.; Pereira, A. Molecular characterization of the CER1 gene of Arabidopsis involved in epicuticular wax biosynthesis and pollen fertility. Plant Cell 1995, 7, 2115-2127. [CrossRef]

8. Brice, B.; Amélie, B.; Frédéric, D.; Stéphanie, P.; Amandine, L.; Dominique, R.; Marjorie, P.; Denis, V.; Haslam, R.P.; Napier, J.A. Overexpression of Arabidopsis ECERIFERUM1 promotes wax very-long-chain alkane biosynthesis and influences plant response to biotic and abiotic stresses. Plant Physiol. 2011, 156, 29-45.

9. Amélie, B.; Frédéric, D.; Stéphanie, P.; Reinhard, J.; Charlotte, R.; Jean-Denis, F.; Haslam, R.P.; Napier, J.A.; René, L.; Jérome, J. Reconstitution of plant alkane biosynthesis in yeast demonstrates that Arabidopsis ECERIFERUM1 and ECERIFERUM3 are core components of a very-long-chain alkane synthesis complex. Plant Cell 2012, 24, 3106-3118.

10. Owen, R.; Huanquan, Z.; Hepworth, S.R.; Patricia, L.; Reinhard, J.; Ljerka, K. CER4 encodes an alcohol-forming fatty acyl-coenzyme A reductase involved in cuticular wax production in Arabidopsis. Plant Physiol. 2006, 142, 866-877.

11. Pierre, B.; Patricia, P.; Erin, O.; CaiZhong, J.; José Luis, R. WIN1, a transcriptional activator of epidermal wax accumulation in Arabidopsis. Proc. Natl. Acad. Sci. USA 2004, 101, 4706-4711.

12. Zhang, J.; Broeckling, C.; Blancaflor, E.; Sledge, M.; Sumner, L.; Wang, Z. Overexpression of WXP1, a putative Medicago truncatula AP2 domain-containing transcription factor gene, increases cuticular wax accumulation and enhances drought tolerance in transgenic alfalfa (Medicago sativa). Plant J. 2010, 42, 689-707. [CrossRef] [PubMed]

13. Youhua, W.; Liyun, W.; Lixia, Z.; Zhijin, Z.; Haiwen, Z.; Ruidang, Q.; Shirong, Z.; Rongfeng, H. An ethylene response factor OsWR1 responsive to drought stress transcriptionally activates wax synthesis related genes and increases wax production in rice. Plant Mol. Biol. 2012, 78, 275-288.

14. Zhou, X.; Jenks, M.A.; Liu, J.; Liu, A.; Zhang, X.; Xiang, J.; Zou, J.; Peng, Y.; Chen, X. Overexpression of transcription factor OsWR2 regulates wax and cutin biosynthesis in rice and enhances its tolerance to water deficit. Plant Mol. Biol. Rep. 2014, 32, 719-731. [CrossRef]

15. Go, Y.S.; Kim, H.; Kim, H.J.; Suh, M.C. Arabidopsis cuticular wax biosynthesis is negatively regulated by the DEWAX gene encoding an AP2/ERF-type transcription factor. Plant Cell 2014, 26, 1666-1680. [CrossRef]

16. Park, C.S.; Go, Y.S.; Suh, M.C. Cuticular wax biosynthesis is positively regulated by WRINKLED4, an AP2/ERF-type transcription factorin Arabidopsis stems. Plant J. 2016, 88, 257-270. [CrossRef]

17. Kim, H.; Go, Y.S.; Suh, M.C. DEWAX2 transcription factor negatively regulates cuticular wax biosynthesis in Arabidopsis leaves. Plant Cell Physiol. 2018, 59, 966-977. 
18. Lam, P.; Zhao, L.; McFarlane, H.E.; Aiga, M.; Lam, V.; Hooker, T.S.; Kunst, L. RDR1 and SGS3, components of RNA-mediated gene silencing, are required for the regulation of cuticular wax biosynthesis in developing inflorescence stems of Arabidopsis. Plant Physiol. 2012, 159, 1385-1395. [CrossRef]

19. Xia, K.; Ou, X.; Gao, C.; Tang, H.; Jia, Y.; Deng, R.; Xu, X.; Zhang, M. OsWS1 involved in cuticular wax biosynthesis is regulated by osa-miR1848. Plant Cell Environ. 2015, 38, 2662-2673. [CrossRef]

20. Lam, P.; Zhao, L.; Eveleigh, N.; Yu, Y.; Chen, X.; Kunst, L. The exosome and trans-acting small interfering RNAs regulate cuticular wax biosynthesis during Arabidopsis inflorescence stem development. Plant Physiol. 2015, 167, 323-336. [CrossRef]

21. Zhao, L.; Kunst, L. SUPERKILLER complex components are required for the RNA exosome-mediated control of cuticular wax biosynthesis in Arabidopsis inflorescence stems. Plant Physiol. 2016, 171, 960-973. [PubMed]

22. Huang, D.; Feurtado, J.A.; Smith, M.A.; Flatman, L.K.; Koh, C.; Cutler, A.J. Long noncoding miRNA gene represses wheat $\beta$-diketone waxes. Proc. Natl. Acad. Sci. USA 2017, 114, E3149-E3158. [CrossRef] [PubMed]

23. Liu, Z.; Fang, Z.; Zhuang, M.; Zhang, Y.; Lv, H.; Liu, Y.; Li, Z.; Sun, P.; Tang, J.; Liu, D. Fine-mapping and analysis of Cgl1, a gene conferring glossy trait in cabbage (Brassica oleracea L. var. capitata). Front. Plant Sci. 2017, 8, 239. [CrossRef] [PubMed]

24. Ji, J.; Cao, W.; Dong, X.; Liu, Z.; Fang, Z.; Zhuang, M.; Zhang, Y.; Lv, H.; Wang, Y.; Sun, P. A 252-bp insertion in BoCER1 is responsible for the glossy phenotype in cabbage (Brassica oleracea L. var. capitata). Mol. Breed. 2018, 38, 128-135. [CrossRef]

25. Liu, D.; Tang, J.; Liu, Z.; Dong, X.; Zhuang, M.; Zhang, Y.; Lv, H.; Sun, P.; Liu, Y.; Li, Z.; et al. Fine mapping of BoGL1, a gene controlling the glossy green trait in cabbage (Brassica oleracea L. var. capitata). Mol. Breed. 2017, 37, 69-77. [CrossRef]

26. Liu, D.; Dong, X.; Liu, Z.; Tang, J.; Zhuang, M.; Zhang, Y.; Lv, H.; Fang, Z.; Yang, L. Fine mapping and candidate gene identification for wax biosynthesis locus, BoWax1 in Brassica oleracea L. var. capitata. Front. Plant Sci. 2018, 9, 309. [CrossRef] [PubMed]

27. Liu, D.; Tang, J.; Liu, Z.; Dong, X.; Zhuang, M.; Zhang, Y.; Lv, H.; Sun, P.; Liu, Y.; Li, Z. Cgl2 plays an essential role in cuticular wax biosynthesis in cabbage (Brassica oleracea L. var. capitata). BMC Plant Biol. 2017, 17, 223. [CrossRef] [PubMed]

28. Ni, E.; Zhou, L.; Li, J.; Jiang, D.; Wang, Z.; Zheng, S.; Qi, H.; Zhou, Y.; Wang, C.; Xiao, S.; et al. OsCER1 plays a pivotal role in very-long-chain alkane biosynthesis and affects plastid development and programmed cell death of tapetum in rice (Oryza sativa L.). Front. Plant Sci. 2018, 9, 1217. [CrossRef] [PubMed]

29. Wang, W.; Zhang, Y.; Xu, C.; Ren, J.; Liu, X.; Black, K.; Gai, X.; Wang, Q.; Ren, H. Cucumber ECERIFERUM1 (CSCER1), which influences the cuticle properties and drought tolerance of cucumber, plays a key role in VLC alkanes biosynthesis. Plant Mol. Biol. 2015, 87, 219-233. [CrossRef]

30. Zhang, J.; Wei, L.; Jiang, J.; Mason, A.S. Genome-wide identification, putative functionality and interactions between lncRNAs and miRNAs in Brassica species. Sci. Rep. 2018, 8, 4960. [CrossRef] [PubMed]

31. Wang, A.; Hu, J.; Gao, C.; Chen, G.; Wang, B.; Lin, C.; Song, L.; Ding, Y.; Zhou, G. Genome-wide analysis of long non-coding RNAs unveils the regulatory roles in the heat tolerance of Chinese cabbage (Brassica rapa ssp. chinensis). Sci. Rep. 2019, 9, 5002. [CrossRef] [PubMed]

32. Huang, L.; Dong, H.; Zhou, D.; Li, M.; Liu, Y.; Zhang, F.; Feng, Y.; Yu, D.; Lin, S.; Cao, J. Systematic identification of long non-coding RNAs during pollen development and fertilization in Brassica rapa. Plant J. 2018, 96, 203-222. [CrossRef] [PubMed]

33. Kornienko, A.E.; Guenzl, P.M.; Barlow, D.P.; Pauler, F.M. Gene regulation by the act of long non-coding RNA transcription. BMC Biol. 2013, 11, 59. [CrossRef] [PubMed]

34. Hannoufa, A.; Mcnevin, J.; Lemieux, B. Epicuticular waxes of eceriferum mutants of Arabidopsis thaliana. Phytochemistry 1993, 33, 851-855. [CrossRef]

35. McNevin, J.P.; Woodward, W.; Hannoufa, A.; Feldmann, K.A.; Lemieux, B. Isolation and characterization of eceriferum (cer) mutants induced by T-DNA insertions in Arabidopsis thaliana. Genome 1993, 36, 610-618. [CrossRef] [PubMed]

36. Jenks, M.A.; Tuttle, H.A.; Eigenbrode, S.D.; Feldmann, K.A. Leaf epicuticular waxes of the eceriferum mutants in Arabidopsis. Plant Physiol. 1995, 108, 369-377. [CrossRef] [PubMed]

37. Vishwanath, S.J.; Kosma, D.K.; Pulsifer, I.P.; Sabine, S.; Stéphanie, P.; Jérome, J.; Franziska, D.D.; René, L.; Owen, R.; Frédéric, D. Suberin-associated fatty alcohols in Arabidopsis: Distributions in roots and contributions to seed coat barrier properties. Plant Physiol. 2013, 163, 1118-1132. [CrossRef] 
38. Lee, S.B.; Jung, S.J.; Go, Y.S.; Kim, H.U.; Kim, J.K.; Cho, H.J.; Park, O.K.; Suh, M.C. Two Arabidopsis 3-ketoacyl CoA synthase genes, KCS20 and KCS2/DAISY, are functionally redundant in cuticular wax and root suberin biosynthesis, but differentially controlled by osmotic stress. Plant J. 2009, 60, 462-475. [CrossRef]

39. Liu, J.; Jung, C.; Xu, J.; Wang, H.; Deng, S.; Bernad, L.; Arenas-Huertero, C.; Chua, N.H. Genome-wide analysis uncovers regulation of long intergenic noncoding RNAs in Arabidopsis. Plant Cell 2012, 24, 4333-4345. [CrossRef]

40. Zhang, Y.C.; Liao, J.Y.; Li, Z.Y.; Yu, Y.; Zhang, J.P.; Li, Q.F.; Qu, L.H.; Shu, W.S.; Chen, Y.Q. Genome-wide screening and functional analysis identify a large number of long noncoding RNAs involved in the sexual reproduction of rice. Genome Biol. 2014, 15, 512. [CrossRef]

41. Li, L.; Eichten, S.R.; Shimizu, R.; Petsch, K.; Yeh, C.T.; Wu, W.; Chettoor, A.M.; Givan, S.A.; Cole, R.A.; Fowler, J.E.; et al. Genome-wide discovery and characterization of maize long non-coding RNAs. Genome Biol. 2014, 15, R40. [CrossRef] [PubMed]

42. Xin, M.; Wang, Y.; Yao, Y.; Song, N.; Hu, Z.; Qin, D.; Xie, C.; Peng, H.; Ni, Z.; Sun, Q. Identification and characterization of wheat long non-protein coding RNAs responsive to powdery mildew infection and heat stress by using microarray analysis and SBS sequencing. BMC Plant Biol. 2011, 11, 61. [CrossRef] [PubMed]

43. Shuai, P.; Liang, D.; Tang, S.; Zhang, Z.; Ye, C.Y.; Su, Y.; Xia, X.; Yin, W. Genome-wide identification and functional prediction of novel and drought-responsive lincRNAs in Populus trichocarpa. J. Exp. Bot. 2014, 65, 4975-4983. [CrossRef] [PubMed]

44. Wang, M.; Yuan, D.; Tu, L.; Gao, W.; He, Y.; Hu, H.; Wang, P.; Liu, N.; Lindsey, K.; Zhang, X. Long noncoding RNAs and their proposed functions in fibre development of cotton (Gossypium spp.). New Phytol. 2015, 207, 1181-1197. [CrossRef] [PubMed]

45. Zhang, C.; Tang, G.; Peng, X.; Sun, F.; Liu, S.; Xi, Y. Long non-coding RNAs of switchgrass (Panicum virgatum L.) in multiple dehydration stresses. BMC Plant Biol. 2018, 18, 79. [CrossRef]

46. Wu, X.; Shi, T.; Iqbal, S.; Zhang, Y.; Liu, L.; Gao, Z. Genome-wide discovery and characterization of flower development related long non-coding RNAs in Prunus mume. BMC Plant Biol. 2019, 19, 64. [CrossRef] [PubMed]

47. Kim, D.; Langmead, B.; Salzberg, S.L. HISAT: A fast spliced aligner with low memory requirements. Nat. Methods 2015, 12, 357-360. [CrossRef]

48. Pertea, M.; Kim, D.; Pertea, G.M.; Leek, J.T.; Salzberg, S.L. Transcript-level expression analysis of RNA-seq experiments with HISAT, StringTie and Ballgown. Nat. Protoc. 2016, 11, 1650-1667. [CrossRef]

49. Kong, L.; Zhang, Y.; Ye, Z.Q.; Liu, X.Q.; Zhao, S.Q.; Wei, L.; Gao, G. CPC: Assess the protein-coding potential of transcripts using sequence features and support vector machine. Nucl. Acids Res. 2007, 35, W345-W349. [CrossRef]

50. Sun, L.; Luo, H.; Bu, D.; Zhao, G.; Yu, K.; Zhang, C.; Liu, Y.; Chen, R.; Zhao, Y. Using sequence intrinsic composition to classify protein-coding and long non-coding transcripts. Nucl. Acids Res. 2013, 41, e166. [CrossRef]

51. Finn, R.D.; Bateman, A.; Clements, J.; Coggill, P.; Eberhardt, R.Y.; Eddy, S.R.; Heger, A.; Hetherington, K.; Holm, L.; Mistry, J.; et al. Pfam: The protein families database. Nucl. Acids Res. 2014, 42, D222-D230. [CrossRef] [PubMed]

52. Wang, L.; Park, H.J.; Dasari, S.; Wang, S.; Kocher, J.P.; Li, W. CPAT: Coding-Potential Assessment Tool using an alignment-free logistic regression model. Nucl. Acids Res. 2013, 41, e74. [CrossRef] [PubMed]

53. Li, J.; Ma, W.; Zeng, P.; Wang, J.; Geng, B.; Yang, J.; Cui, Q. LncTar: A tool for predicting the RNA targets of long noncoding RNAs. Brief. Bioinform. 2015, 16, 806-812. [CrossRef] [PubMed]

54. Mao, X.; Cai, T.; Olyarchuk, J.G.; Wei, L. Automated genome annotation and pathway identification using the KEGG Orthology (KO) as a controlled vocabulary. Bioinformatics 2005, 21, 3787-3793. [CrossRef] [PubMed]

55. Wu, J.; Mao, X.; Cai, T.; Luo, J.; Wei, L. KOBAS server: A web-based platform for automated annotation and pathway identification. Nucl. Acids Res. 2006, 34, W720-W724. [CrossRef] [PubMed]

(C) 2019 by the authors. Licensee MDPI, Basel, Switzerland. This article is an open access article distributed under the terms and conditions of the Creative Commons Attribution (CC BY) license (http://creativecommons.org/licenses/by/4.0/). 